\title{
Efficacy of Routine Fecal Microbiota Transplantation for Treatment of Recurrent Clostridium difficile Infection: A Retrospective Cohort Study
}

\author{
Alexandra Nowak, ${ }^{1}$ Magnus Hedenstierna, ${ }^{2}$ Johan Ursing, ${ }^{2,3}$ Christer Lidman, \\ and Piotr Nowak iD ${ }^{4}$ \\ ${ }^{1}$ Department of Nephrology, Karolinska University Hospital Huddinge, Huddinge, Sweden \\ ${ }^{2}$ Department of Infectious Diseases, Danderyds Hospital, Danderyd, Sweden \\ ${ }^{3}$ Department of Clinical Sciences, Danderyds Hospital, Danderyd, Sweden \\ ${ }^{4}$ Department of Medicin Huddinge, Unit of Infectious Diseases, Karolinska Institutet, Karolinska University Hospital, \\ Huddinge, Sweden
}

Correspondence should be addressed to Piotr Nowak; piotr.nowak@ki.se

Received 5 April 2019; Revised 31 May 2019; Accepted 2 June 2019; Published 1 July 2019

Academic Editor: Joseph Falkinham

Copyright ( 2019 Alexandra Nowak et al. This is an open access article distributed under the Creative Commons Attribution License, which permits unrestricted use, distribution, and reproduction in any medium, provided the original work is properly cited.

\begin{abstract}
Background. Patients with recurrent Clostridium difficile infections (CDIs) constitute an increasing treatment problem. Fecal microbiota transplantation (FMT) has shown promising results of treating recurrent CDI, where treatment with antibiotics fails repeatedly. Our study describes retrospective cohort treated with FMT at two major hospitals in Stockholm. Methods. Medical records of all patients with recurrent CDI treated with FMT during the period 2013-2017 were reviewed. We evaluated cure of CDI-related diarrhea without relapse 10 weeks after FMT. Results. 47 patients were included. One treatment cured 25 patients (53\%), and more than one treatment cured 32 patients (68\%). Treatment outcome did not vary significantly with treatment with fresh donor feces or frozen fecal culture, days of use of antibiotics or days of hospitalization prior to CDI, and renal function or time from the first CDI to therapy. Treatment failure was associated with a significantly lower Karnofsky performance status score (70 points vs $90, p=0.02$ ). Conclusion. Fecal instillation, for the treatment of relapsing CDI, is a promising approach, with $68 \%$ success rate reported in this study. The success rate of FMT is high, regardless of multiple comorbidities, extended use of antibiotics, or long time hospitalization. Although generally FMT is performed with fresh donor feces, our data show that the usage of frozen fecal culture could be an effective treatment alternative in recurrent CDI.
\end{abstract}

\section{Introduction}

Clostridium difficile is an anaerobic Gram-positive, sporeforming, toxin-producing bacillus that is transmitted among humans through the fecal-oral route. C. difficile spores resistant to heat, acid, and antibiotics are common in health care facilities and low levels are found in the environment and food, allowing for nosocomial and community transmission [1].

In the United States, C. difficile is the most frequently reported nosocomial pathogen. A surveillance study in 2011 identified 453,000 cases of cases of $C$. difficile infection (CDI) and 29,000 deaths associated with CDI [1]. The Public Health Agency of Sweden estimates that there are approximately 400 cases of recurrent CDI/year in Stockholm area (almost 1100 cases/year of CDI in total) [2]. Infection is prevented by barrier properties of the fecal microbiota. Weakening of this defense by antibiotic treatment is the major risk factor for symptomatic $C$. difficile infection. Additionally, advanced age, use of proton pump inhibitors (PPI), and multiple comorbidities such as chronic heart and lung diseases, kidney failure, and diabetes also contribute to CDI susceptibility. However, symptoms of colitis do not develop in all colonized persons $[1,3]$. 
According to the European Society of Clinical Microbiology and Infectious Diseases guidelines, first-line treatment of CDI includes either metronidazole or vancomycin [4]. CDI relapses in approximately $15-26 \%$ of patients treated for a first episode [5]. Treatment of a second or later recurrence commonly includes tapered or long-term vancomycin but is even less effective with over $50 \%$ risk for continued recurrence $[5,6]$. Due to high recurrence rates and alteration of colonic microbiota with the continued use of antimicrobial drugs, new approaches in CDI therapy include new narrow-spectrum antibiotics, probiotics, and monoclonal antibodies $[7,8]$. For example, fidaxomicin has been shown to reduce the rate of recurrence compared with vancomycin; however, its use is limited by costs [9].

A promising way of treating recurrent CDI, which has attracted much interest in recent years, is reintroduction of normal intestinal flora by infusion of feces from healthy donors or a stool substitute obtained from purified fecal cultures [10]. Restoring normal colon microbiota was first described by Eiseman and coworkers in 1958 [11]. Reports from case series and meta-analyses show success rates from 64-95\% [12-15].

Fecal instillation with donated feces or purified feces culture has been used to routinely treat patients with multiple CDI recurrences at the Departments of Infectious Diseases at Danderyd Hospital and Karolinska University Hospital Huddinge in Stockholm/Sweden for many years. However, FMT has been reserved to treat the most difficult cases. This work presents the results of all 47 patients with recurrent CDI, treated with fecal instillation in the period 2013-2017.

\section{Materials and Methods}

2.1. Study Design. This was a retrospective analysis of the characteristics and outcomes of all patients with recurrent CDI treated in the period from September 2013 to September 2017.

2.2. Study Sites. The study was conducted at the departments of infectious diseases at Danderyd Hospital and Karolinska University Hospital, Huddinge. The sites are two of Stockholm's major hospitals where FMT is done.

2.3. Data Collection. We examined electronic medical records for patients using a predefined data entry form that included information on clinical outcome 10 weeks after FMT. We also collected data on hospitalization 90 days prior to CDI and antimicrobial drug treatment 90 days prior to CDI.

Comorbidities were analyzed using the Charlson Comorbidity Index [16] which predicts the one-year mortality for a patient who may have a range of comorbid conditions such as heart and lung disease, dysfunction of the liver and kidney, and prior cerebral-vascular events diabetes or cancer (a total of 22 conditions). Each condition was assigned a score of $1,2,3$, or 6 , depending on the mortality risk associated with each one. Scores were summed to provide a total score to predict mortality. We also evaluated the Karnofsky Performance Scale Index [17], an estimate of the patient's overall performance status or ability to perform their activities of daily living. It is a single score between 10 and 100 assigned by a clinician based on observations of a patient's ability to perform common tasks relating to activity, work, and self-care. A score of 100 signifies normal physical abilities with no evidence of disease. Decreasing numbers indicate a reduced performance. In the study, we also recorded the use of proton pump inhibitors (PPI), renal function at CDI diagnosis, and days from first CDI to FMT. Additionally, we have collected the data on number of days to CDI recurrence, adverse events up to 10 weeks after FMT, and mortality 12 months after FMT.

2.4. Definitions. CDI was defined as the presence of unformed frequent stools (at least 3/day) for the last 48 hours and a positive PCR test for $C$. difficile toxin B DNA (Karolinska Microbiology Laboratory). Treatment success was defined as clinical cure 10 weeks after FMT $[18,19]$. Individuals with a positive stool sample for $C$. difficile toxin $B$ DNA during follow-up but no diarrhea were classified as a treatment success. Treatment failure was defined as recurrent diarrhea where the physician in charge decided to treat the patient for suspected or confirmed C. difficile. Adverse events were elicited by us for a modification of the Common Terminology Criteria for Adverse Events version 4.0 [20].

2.5. Feces Handling. Donors were healthy relatives or close contacts (without chronic diseases, diarrhea, and antibiotics usage within last two months). They were screened with serology for HIV, HBV, and HCV and by culturing of fecal samples and or PCRs for resistant Enterococcus (VRE), extended-spectrum beta lactamase (ESBL), C. difficile, Salmonella, Shigella, and Campylobacter. Donor feces were delivered to the hospital within 24 hours of evacuation in a clean, closed plastic container. The donor feces were refrigerated until instillation, at the day of transplant. Approximately two table spoons of donor feces (corresponding to $30 \mathrm{~g}$ of feces) were diluted with $500 \mathrm{ml}$ room temperate 0 , 9\% sodium chloride solution, and blended into a homogenous liquid in an electric blender.

The feces culture originates from 1994 and was obtained from a feces sample of healthy Scandinavian donor on ordinary Western diet as previously described [21]. The culture $(30 \mathrm{ml})$ was stored at $-70^{\circ} \mathrm{C}$ at the Department of Microbiology, Tumor and Cell biology Karolinska Institute and delivered to the hospital the day before transplant. The culture was thawed in room temperature $1 \mathrm{~h}$ prior to transplant.

2.6. Fecal Microbiota Transplantation Procedure. Patients were requested to cease treatment with vancomycin or metronidazole at least $24 \mathrm{~h}$ prior to instillation procedure. All patients received loperamide $1 \mathrm{~h}$ prior to the procedure to promote retention of the FMT. Rectal administration was 
performed in most of the patients ( 42 out of 47 ) via the rectal catheter during $30 \mathrm{~min}$ with the patient recumbent on the left side. In five cases, FMT was installed through a nasogastric tube. Patients were bedbound $1 \mathrm{~h}$ after the procedure and informed not to eat two hours after instillation.

2.7. Ethical Approval. Regional Ethics Committee Stockholm has reviewed and approved the study (dnr 2018/54-31/ 2).

2.8. Data Management and Statistical Analysis. The data are presented as median (range) as indicated. Nonparametric statistical analyses were applied with the Fisher exact test for the categorical and Mann-Whitney test for continuous variables, respectively. A $p$ value $<0.05$ was considered as significant. All statistical analyses were performed in GraphPad Prism v 6.0.

\section{Results}

3.1. Study Population. During the study period (September 24,2013 , to September 7, 2017), a total of 48 patients with CDI were treated with FMT at Danderyd Hospital $(n=28)$ and Karolinska University Hospital, Huddinge $(n=20)$. One patient was excluded from the study due to lack of follow-up 10 weeks after FMT as the patient died due to severe lung fibrosis, not related to FMT (3 weeks after FMT). We have thus analyzed 47 patient records during the study period.

Thirty-three (62\%) patients were women. Median age of the cohort was 70 years (range 25-95 years). Thirty-four patients had at least three recurrent CDI episodes prior to FMT. All patients had failed prior vancomycin tapering therapy. Of the 47 patients included in the study, eight patients (17\%) had no use of antibiotics 90 days prior to the first episode of CDI and 12 patients (26\%) were prescribed one antibiotic regime 90 days prior to CDI (Table 1).

Thirty-three (70\%) patients received donor feces, and 14 (30\%) patients received feces culture. There were no major adverse effects reported due to FMT. However, seven patients reported abdominal pain and/or flatulence during or immediately after FMT. One patient was admitted to hospital two days after FMT due to high fever and worsening of symptoms related to CDI. Three patients (6\%) died during the 12 -month follow-up period due to non-CDI/FMTrelated causes.

3.2. Effects of FMT. Twenty-five patients (53\%) were cured after one treatment. Seven additional patients were cured after 2-4 FMTs (Figure 1(a)), resulting in an overall cure rate of $68 \%$. No significant difference was found between male and female patients, with cure rates of $12(86 \%)$ of male and $20(60 \%)$ of female patients, respectively.

In total, $23(72 \%)$ patients who received donor feces and nine $(64 \%)$ patients who received fecal culture were cured. Nineteen patients (57\%) who received donor feces and six patients $(43 \%)$ who received bacterial culture were cured after first FMT. CDI relapsed within three weeks after the procedure (median 7 days, range 1-45 days) in majority of patients in whom FMT failed (86\%). The statistical analyses did not reveal any significant associations between cure rate and age, gender, or mode of FMT (donor vs cultures).

Seven of eight patients $(87 \%)$ who had not been prescribed antibiotics 90 days prior to the first CDI were cured after FMT and 25 out of 39 patients (64\%) who had been prescribed one or more antibiotic regimes within 90 days prior to CDI were cured (Figure $1(\mathrm{~b})$ ). The FMT cure rate was 60 and $65 \%$ in patients on short (1-10 days) and long (>11 days) antibiotic treatment (within 90 days prior to $\mathrm{CDI})$, respectively.

The Charlson Comorbidity Index in the cohort was the same in the cured and non-cured groups (2 range 0-7), indicating a one-year mortality of $10 \%$. Prior to FMT, 18 patients $(38 \%)$ had an eGFR $<60$. However, impaired renal function was not associated with the effectiveness of FMT (Figure 1(c)). The median Karnofsky performance score was significantly higher in the cured group compared to patients who had failed the FMT (median; 90 vs $70 ; p=0.02$ ) (Figure 1(d)). There was no difference in cure rate with regards to numbers of days to first FMT, number of comorbidities, GI surgery, PPI usage, and hospitalization 90 days prior to CDI.

\section{Discussion}

This retrospective study summarizes the outcome of FMT in 47 patients with recurrent CDI in routine clinical settings. FMT was a well-tolerated and effective treatment for CDI, with an overall success rate of $68 \%$. Additionally, we report equal FMT efficacy using fresh donor stools as compared to frozen fecal cultures. The study highlights that FMT has a higher success rate in patients with a higher Karnofsky performance score indicating a generally healthier individual. Despite that, no association between cure rate and several factors previously described as associated with CDI treatment failure such as number of comorbidities, renal failure, days to FMT, and duration of antibiotics treatment was found. [1, 3] The high treatment success rate and relatively small number of patients may account for this lack of association. Nevertheless, an important overall observation in our study is that FMT has a high success rate regardless of age and comorbidities.

Higher FMT efficacy has been described in some but not all earlier studies $[5,10,12,21]$. For instance, several studies reported overall cure rate of $80-95 \%[13,22]$, while others showed success rates of $64-69 \%[12,23]$, i.e., similar to the results presented here. We could not identify differences in baseline demographic or clinical characteristics of the patients in our study and the studies with higher FMT success rate. The difference in cure rate may depend on the dosage of fecal material as well as FMT procedures which are not standardized throughout studies. A single dose of FMT typically uses $50 \mathrm{~g}$ of fecal material, but a different amount of feces have been used ranging from 10-100 $\mathrm{g}$ of fecal material per dose [23-25]. According to the FMT protocol routinely applied in two study centers in Stockholm, approximately 
TABLE 1: Clinical characteristics of the patients.

\begin{tabular}{|c|c|c|c|c|}
\hline & All & Success & Failure & $p$ value \\
\hline Number of patients & 47 & 32 & 15 & - \\
\hline \multicolumn{5}{|l|}{ Age } \\
\hline Median, years (range) & $70(25-95)$ & $69.5(25-90)$ & $74(40-95)$ & n.s. \\
\hline \multicolumn{5}{|l|}{ Gender } \\
\hline Female, $n(\%)$ & $33(70)$ & $20(61)$ & $13(39)$ & \multirow{2}{*}{ n.s. } \\
\hline Male, $n(\%)$ & $14(30)$ & $12(86)$ & $2(14)$ & \\
\hline \multicolumn{5}{|l|}{ Recurrent CDI, $n(\%)$} \\
\hline $1-2$ & $13(27)$ & $9(28)$ & $4(26)$ & \multirow{2}{*}{ n.s. } \\
\hline 3 or more & $34(73)$ & $23(72)$ & $11(74)$ & \\
\hline \multicolumn{5}{|c|}{$\begin{array}{l}\text { Antibiotics regimes } 90 \text { days } \\
\text { prior to the first CDI }\end{array}$} \\
\hline No, $n(\%)$ & $8(17)$ & $7(87.5)$ & $1(12.5)$ & \multirow{2}{*}{ n.s. } \\
\hline Yes, $n(\%)$ & $39(83)$ & $25(64)$ & $14(36)$ & \\
\hline \multicolumn{5}{|l|}{$\begin{array}{l}\text { Days of antibiotics } 90 \text { days } \\
\text { prior to CDI* }\end{array}$} \\
\hline $0(\%)$ & $8(17)$ & $7(87.5)$ & $1(12.5)$ & \multirow{3}{*}{ n.s. } \\
\hline $1-10(\%)$ & $15(33)$ & $9(60)$ & $6(40)$ & \\
\hline$>11-20(\%)$ & $23(50)$ & $15(65)$ & $8(35)$ & \\
\hline \multicolumn{5}{|c|}{$\begin{array}{l}\text { Reported use of PPI at CDI } \\
\text { diagnosis }(\%)\end{array}$} \\
\hline Yes & $25(53)$ & $16(64)$ & $9(36)$ & \multirow{2}{*}{ n.s. } \\
\hline No & $22(47)$ & $16(73)$ & $6(27)$ & \\
\hline \multicolumn{5}{|c|}{$\begin{array}{l}\text { Days of hospitalization } 90 \text { days } \\
\text { prior to CDI* }\end{array}$} \\
\hline $0(\%)$ & $24(52)$ & $17(71)$ & $7(29)$ & \multirow{3}{*}{ - } \\
\hline $1-10(\%)$ & $10(22)$ & $7(70)$ & $3(30)$ & \\
\hline$>11-20(\%)$ & $12(26)$ & $7(58)$ & $5(42)$ & \\
\hline \multicolumn{5}{|c|}{ Known GI surgery prior to CDI (\%) } \\
\hline Yes & $16(34)$ & $13(81)$ & $3(19)$ & \multirow{2}{*}{ - } \\
\hline No & $31(66)$ & $19(61)$ & $12(39)$ & \\
\hline \multicolumn{5}{|c|}{ Kidney function at CDI (eGFR), $n(\%)$} \\
\hline$>90$ & $10(22)$ & $9(90)$ & $1(10)$ & \multirow{3}{*}{-} \\
\hline $60-89$ & $19(40)$ & $12(63)$ & $7(37)$ & \\
\hline $\mathrm{HD}$ and $\mathrm{PD}<15-59$ & $18(38)$ & $11(61)$ & $7(39)$ & \\
\hline \multicolumn{5}{|l|}{ Feces type (\%) } \\
\hline Culture no. & $14(30)$ & $9(64)$ & $5(36)$ & \multirow{2}{*}{ - } \\
\hline Donor no. & $33(70)$ & $23(70)$ & $10(30)$ & \\
\hline \multicolumn{5}{|c|}{ Karnofsky performance status } \\
\hline Median (range) & $80(40-100)$ & $90(50-100)$ & $70(40-100)$ & $p=0.02$ \\
\hline \multicolumn{5}{|l|}{ No. of comorbidities (\%) } \\
\hline $0-1$ & $14(30)$ & $11(78)$ & $3(21)$ & \multirow{3}{*}{-} \\
\hline $2-3$ & $23(49)$ & $14(61)$ & $9(39)$ & \\
\hline$>4$ & $10(21)$ & $7(70)$ & $3(30)$ & \\
\hline \multicolumn{5}{|l|}{ Days to first FMT } \\
\hline Median & 144 & 155.5 & 138 & \multirow[b]{2}{*}{ - } \\
\hline Range & $9-884$ & $9-884$ & $40-337$ & \\
\hline
\end{tabular}

${ }^{*}$ Data based on 46 of 47 individuals; $p$ value $<0.05$ was considered as significant; n.s.: not significant.

$30 \mathrm{~g}$ of fecal material was administered which may have affected the outcome in some patients. We cannot explain the lower cure rate with the choice of administrated material as we found no difference in the outcome of FMT regarding usage of fresh donor feces or frozen fecal cultures. Even if not fully applicable to our situation, a recent meta-analysis by Tang et al. [26] also indicates that the treatment efficacy of frozen FMT and fresh FMT was similar. A possible contributing factor to the lower efficacy might be selection of the most difficult to cure patients in this study as FMT in our setting was reserved for this patient group.

Additionally, some reports suggest that patients with recurrent CDI may benefit from multiple serial FMT procedures $[14,15,27]$. In the two Stockholm centers, single FMT procedures were routinely conducted, and in our study, we observed $10 \%$ increase of cure rate with additional FMT. However, as highlighted by Kelly and Tebas [28], much work remains to be done in defining the optimal FMT dose, 


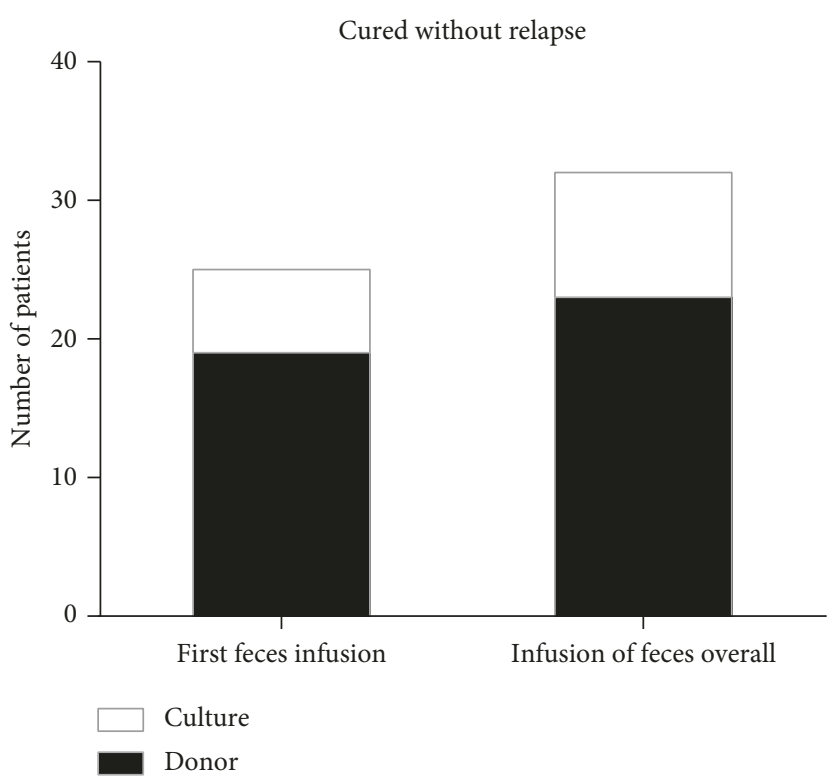

(a)

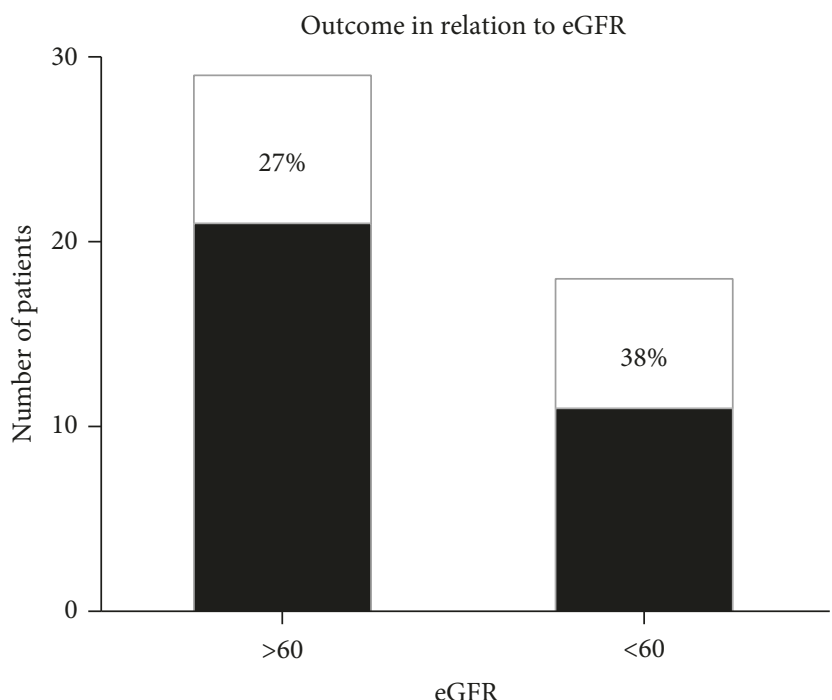

eGFR

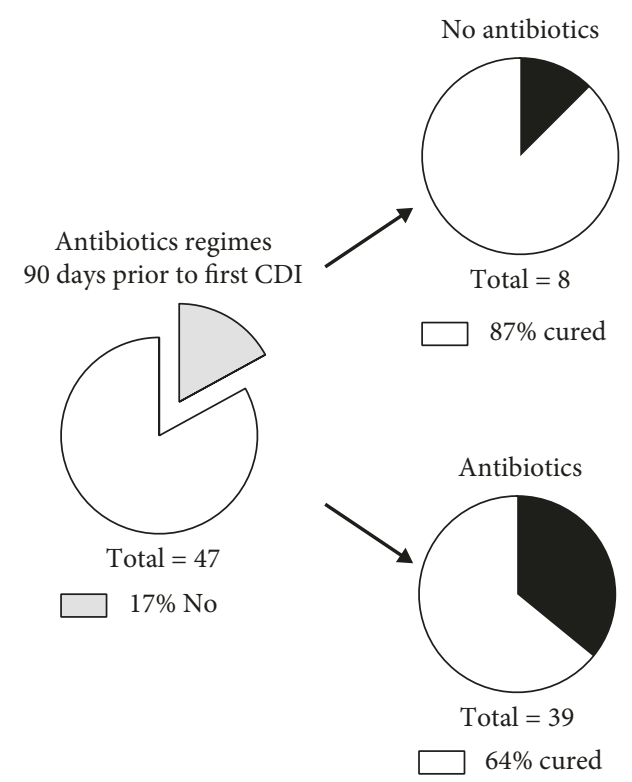

(b)

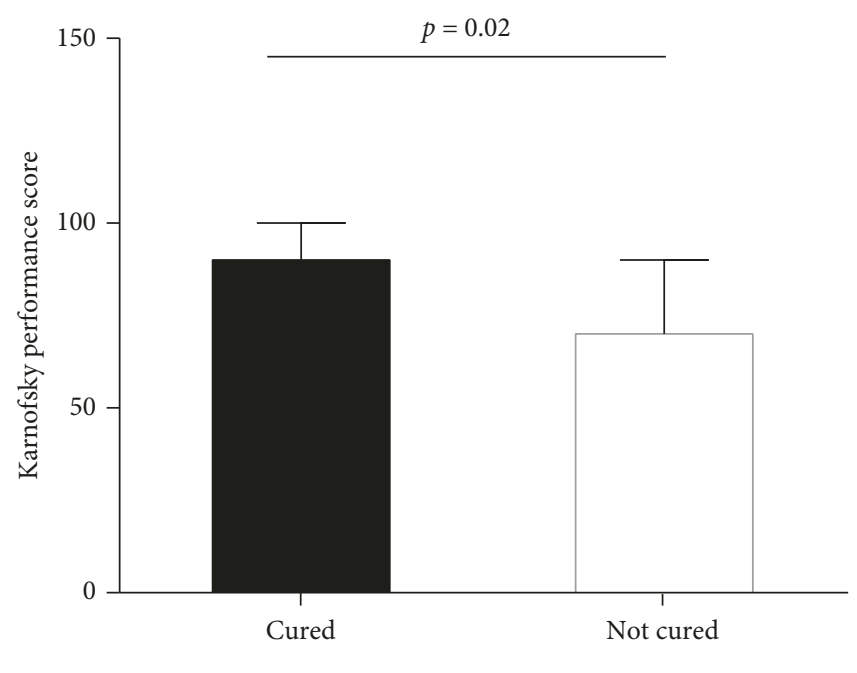

(d)

Figure 1: Outcome of fecal microbial transplantation in the cohort in relation to several factors: number of FMT treatments with donor feces or fecal culture (a); intake of antibiotics 90 days prior to C. difficile infection (b); eGFR (c); Karnofsky performance score (d).

recipient preparation, and post-FMT monitoring. In light of this, it is worthwhile noting that the fecal culture used in this study appeared to be as effective as freshly donated fecal material. This suggests that it is possible to establish one or perhaps several defined microbiota cultures that may enable standardized FMT in addition to having significant practical and regulatory implications.

Of the 47 patients included in the study, eight patients (17\%) had no use of antibiotics 90 days prior to the first episode of CDI and 12 patients (26\%) were prescribed one antibiotic regime 90 days prior to CDI. This illustrates that CDI in our cohort was not solely affecting individuals with repeated and extended antibiotic treatment and that a significant amount of CDI is community acquired as previously described [1].

Recovery of the intestinal flora is believed to be behind cure with FMT but the mechanism of FMT effect is still not fully resolved. It remains to be understood whether reconstituting the bacterial community alone or support from other microbes and/or metabolites play a role in the resolution [25]. Nevertheless, FMT is emerging as an effective therapy for recurrent CDI, and it is being investigated as a treatment option for other inflammatory conditions. More recently, clinical trials have focused on the role of FMT 
in inflammatory bowel disease (IBD) [29]. Whether FMT aids IBD control or provokes IBD exacerbation is an area of active debate, and at present, no conclusive statement can be made on the balance between FMT's benefits and risks in this population. Published case reports have also demonstrated clinical improvement after FMT in conditions not classically associated with gastrointestinal diseases such as multiple sclerosis, Parkinson's disease, alopecia areata, and idiopathic thrombocytopenic purpura [30-32]. This suggests not only a local intestinal effect but a profound immunological response to FMT. As such, more research is needed to explain mechanism of FMT and its immunological effects. Additionally, the long-term effects of microbiota manipulation are still unclear, especially in light of the increasingly recognized roles of the human gut microbiome in health and disease [33].

Knowing its limitations, we have to bear in mind that the FMT has been used for decades and has a definite place in treatment of the recurrent CDI. The results from our retrospective study in clinical settings fully support the future use of FMT in this patients group as safe procedure to cure CDI regardless of patients' comorbidities. Although generally the FMT is performed using the fresh or frozen donor stools, we propose that the usage of frozen fecal culture may be a fully effective alternative in recurrent CDI.

\section{Data Availability}

The clinical data used to support the findings of this study are available from the corresponding author upon request.

\section{Conflicts of Interest}

The authors declare that there are no conflicts of interest.

\section{Acknowledgments}

This work was supported by the Åke Wiberg Foundation grant (given to PN).

\section{References}

[1] D. A. Leffler and J. T. Lamont, "Clostridium difficile infection," New England Journal of Medicine, vol. 372, no. 16, pp. 1539-1548, 2015.

[2] Clostridium Difficile Infection-Swedish Guidance for Surveillance, Prevention and Outbreak Management, https:// www.folkhalsomyndigheten.se.

[3] N. Bagdasarian, K. Rao, and P. N. Malani, "Diagnosis and treatment of Clostridium difficile in adults," JAMA, vol. 313, no. 4, pp. 398-408, 2015.

[4] S. B. Debast, M. P. Bauer, and E. J. Kuijper, "European society of clinical microbiology and infectious diseases: update of the treatment guidance document for Clostridium difficile infection," Clinical Microbiology and Infection, vol. 20, no. 2, pp. 1-26, 2014.

[5] L. V. McFarland, C. M. Surawicz, M. Rubin, R. Fekety, G. W. Elmer, and R. N. Greenberg, "Recurrent Clostridium difficile disease: epidemiology and clinical characteristics," Infection Control \& Hospital Epidemiology, vol. 20, no. 1, pp. 43-50, 1999.
[6] R. Fekety, L. V. McFarland, C. M. Surawicz, R. N. Greenberg, G. W. Elmer, and M. E. Mulligan, "Recurrent Clostridium difficile diarrhea: characteristics of and risk factors for patients enrolled in a prospective, randomized, double-blinded trial," Clinical Infectious Diseases, vol. 24, no. 3, pp. 324-333, 1997.

[7] C. M. F. van Nispen tot Pannerden, A. Verbon, and E. J. Kuipers, "Recurrent Clostridium difficile infection," Drugs, vol. 71, no. 7, pp. 853-868, 2011.

[8] D. N. Gerding and S. Johnson, "Management of Clostridium difficile infection: thinking inside and outside the box," Clinical Infectious Diseases, vol. 51, no. 11, pp. 1306-1313, 2010.

[9] S. M. Bartsch, C. A. Umscheid, N. Fishman, and B. Y. Lee, "Is fidaxomicin worth the cost? An economic analysis," Clinical Infectious Diseases, vol. 57, no. 4, pp. 555-561, 2013.

[10] N. A. Cohen, D. M. Livovsky, S. Yaakobovitch et al., “A retrospective comparison of fecal microbial transplantation methods for recurrent Clostridium difficile infection," Israel Medical Association Journal, vol. 18, no. 10, pp. 594-599, 2016.

[11] B. Eiseman, W. Silen, G. S. Bascom, and A. J. Kauvar, "Fecal enema as an adjunct in the treatment of pseudomembranous enterocolitis," Surgery, vol. 44, no. 5, pp. 854-859, 1958.

[12] M. Tvede, M. Tinggaard, and M. Helms, "Rectal bacteriotherapy for recurrent Clostridium difficile-associated diarrhoea: results from a case series of 55 patients in Denmark 2000-2012," Clinical Microbiology and Infection, vol. 21, no. 1, pp. 48-53, 2015.

[13] C. H. Lee, J. E. Belanger, Z. Kassam et al., "The outcome and long-term follow-up of 94 patients with recurrent and refractory Clostridium difficile infection using single to multiple fecal microbiota transplantation via retention enema," $E \mathbf{u}$ ropean Journal of Clinical Microbiology \& Infectious Diseases, vol. 33, no. 8, pp. 1425-1428, 2014.

[14] M. N. Quraishi, M. Widlak, N. Bhala et al., "Systematic review with meta-analysis: the efficacy of faecal microbiota transplantation for the treatment of recurrent and refractory Clostridium difficile infection," Alimentary Pharmacology \& Therapeutics, vol. 46, no. 5, pp. 479-493, 2017.

[15] G. Ianiro, M. Maida, J. Burisch et al., "Efficacy of different faecal microbiota transplantation protocols for Clostridium difficile infection: a systematic review and meta-analysis," United European Gastroenterology Journal, vol. 6, no. 8, pp. 1232-1244, 2018.

[16] M. Charlson, T. P. Szatrowski, J. Peterson, and J. Gold, "Validation of a combined comorbidity index," Journal of Clinical Epidemiology, vol. 47, no. 11, pp. 1245-1251, 1994.

[17] V. Crooks, S. Waller, T. Smith, and T. J. Hahn, "The use of the Karnofsky Performance Scale in determining outcomes and risk in geriatric outpatients," Journal of Gerontology, vol. 46, no. 4, pp. M139-M144, 1991.

[18] E. van Nood, P. Speelman, E. J. Kuijper, and J. J. Keller, "Struggling with recurrent Clostridium difficile infections: is donor faeces the solution?," Eurosurveillance, vol. 14, no. 34, 2009.

[19] C. M. Surawicz, L. J. Brandt, D. G. Binion et al., "Guidelines for diagnosis, treatment and prevention of Clostridium difficile infections," American Journal of Gastroenterology, vol. 108, no. 4, pp. 478-498, 2013.

[20] Common Terminology Criteria for adverse events (CTCAE) version 4.0 published: May 28, 2009 (v4.03: June 14, 2010), https://evs.nci.nih.gov/.

[21] A. Gustafsson, S. Lund-Tønnesen, A. Berstad, T. Midtvedt, and E. Norin, "Faecal short-chain fatty acids in patients with 
antibiotic-associated diarrhoea, before and after faecal enema treatment," Scandinavian Journal of Gastroenterology, vol. 33, no. 7, pp. 721-727, 1998.

[22] H. Zainah, M. Hassan, L. Shiekh-Sroujieh, S. Hassan, G. Alangaden, and M. Ramesh, "Intestinal microbiota transplantation, a simple and effective treatment for severe and refractory Clostridium difficile infection," Digestive Diseases and Sciences, vol. 60, no. 1, pp. 181-185, 2015.

[23] C. Jorup-Rönström, A. Håkanson, S. Sandell et al., "Fecal transplant against relapsing Clostridium difficile-associated diarrhea in 32 patients," Scandinavian Journal of Gastroenterology, vol. 47, no. 5, pp. 548-552, 2012.

[24] Z. Kasssam, C. H. Lee, Y. Yuan, and R. H. Junt, "Fecal microbiota transplantation for Clostridium difficile infections: systematic review and meta-analysis," American Journal of Gastroenterology, vol. 108, no. 4, pp. 500-508, 2013.

[25] G. Cammarota, G. Ianiro, and A. Gasbarrini, "Fecal microbiota transplantation for the treatment of Clostridium difficile infection," Journal of Clinical Gastroenterology, vol. 48, no. 8, pp. 693-702, 2014.

[26] G. Tang, W. Yin, and W. Liu, "Is frozen fecal microbiota transplantation as effective as fresh fecal microbiota transplantation in patients with recurrent or refractory Clostridium difficile infection: a meta-analysis?," Diagnostic Microbiology and Infectious Disease, vol. 88, no. 4, pp. 322-329, 2017.

[27] M. Fischer, B. W. Sipe, N. A. Rogers et al., "Faecal microbiota transplantation plus selected use of vancomycin for severecomplicated Clostridium difficile infection: description of a protocol with high success rate," Alimentary Pharmacology \& Therapeutics, vol. 42, no. 4, pp. 470-476, 2015.

[28] B. J. Kelly and P. Tebas, "Clinical practice and infrastructure review of fecal microbiota transplantation for Clostridium difficile infection," Chest, vol. 153, no. 1, pp. 266-277, 2018.

[29] T. J. Borody, S. Paramsothy, and G. Agrawal, "Fecal microbiota transplantation: indications, methods, evidence, and future directions," Current Gastroenterology Reports, vol. 15, no. 8 , p. 337, 2013

[30] D. Rebello, E. Wang, E. Yen, P. A. Lio, and C. R. Kelly, "Hair growth in two alopecia patients after fecal microbiota transplant," ACG Case Reports Journal, vol. 4, no. 1, p. e107, 2017.

[31] C. R. Camara-Lemarroy, L. M. Metz, and V. W. Yong, "Focus on the gut-brain axis: multiple sclerosis, the intestinal barrier and the microbiome," World Journal of Gastroenterology, vol. 24, no. 37, pp. 4217-4223, 2018.

[32] M.-F. Sun and Y.-Q. Shen, "Dysbiosis of gut microbiota and microbial metabolites in Parkinson's Disease," Ageing Research Reviews, vol. 45, pp. 53-61, 2018.

[33] R. E. Ooijevaar, E. M. Terveer, H. W. Verspaget, E. J. Kuijper, and J. J. Keller, "Clinical application and potential of fecal microbiota transplantation," Annual Review of Medicine, vol. 70, no. 1, pp. 335-351, 2018. 


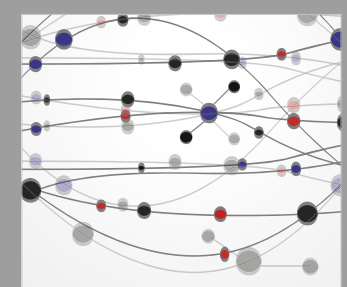

The Scientific World Journal
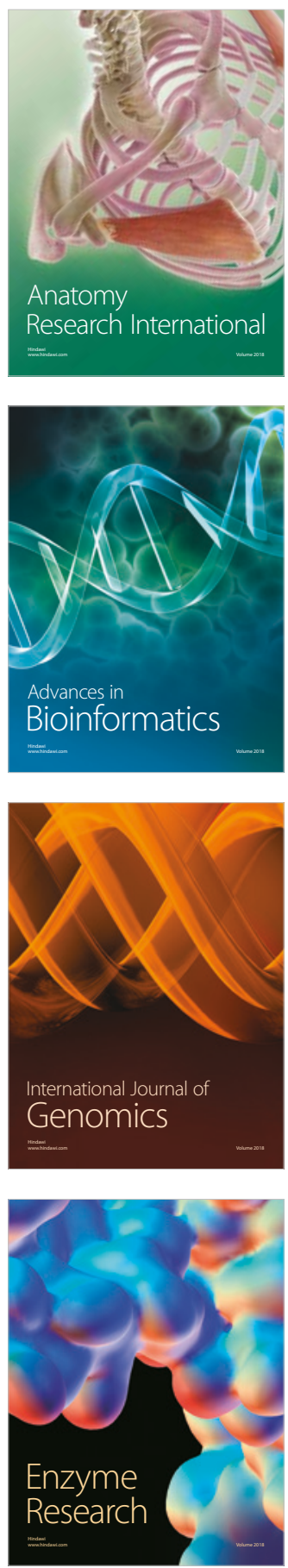
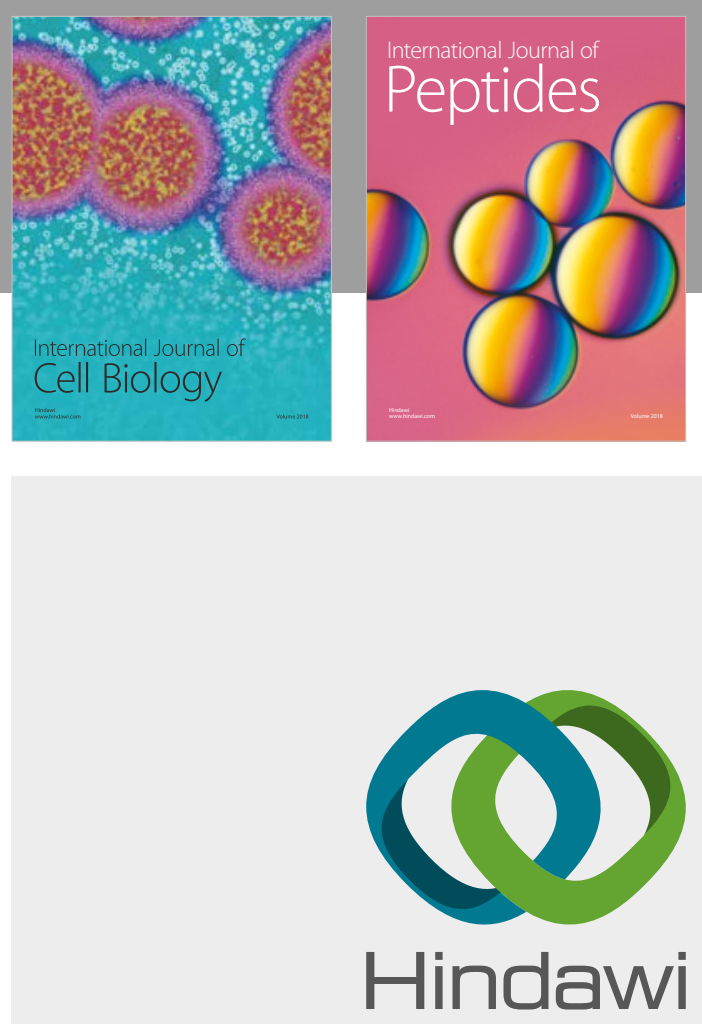

Submit your manuscripts at

www.hindawi.com
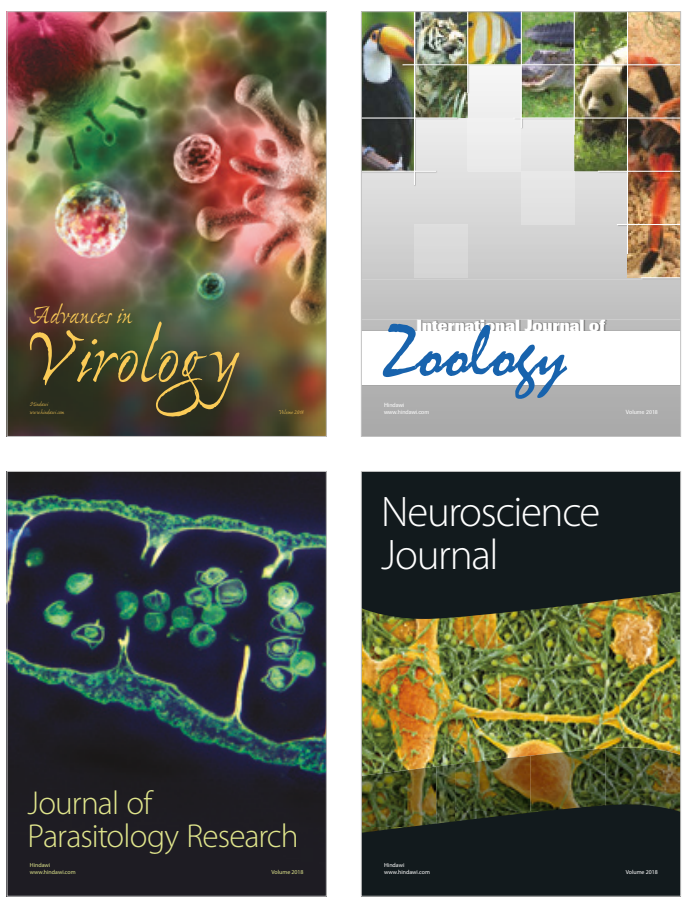
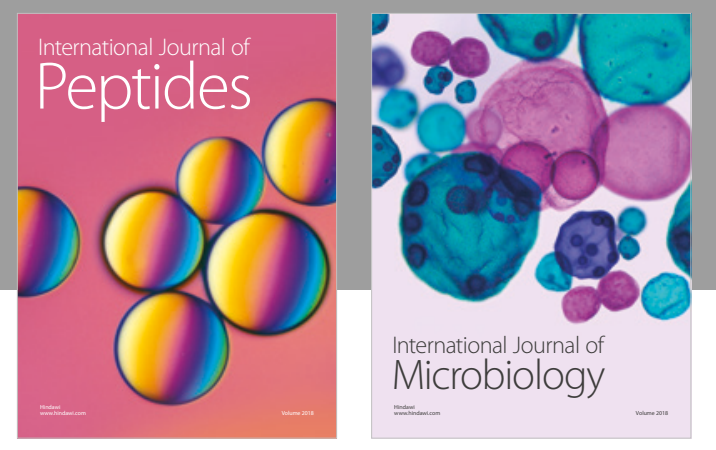

nternational Journal of Microbiology
Journal of
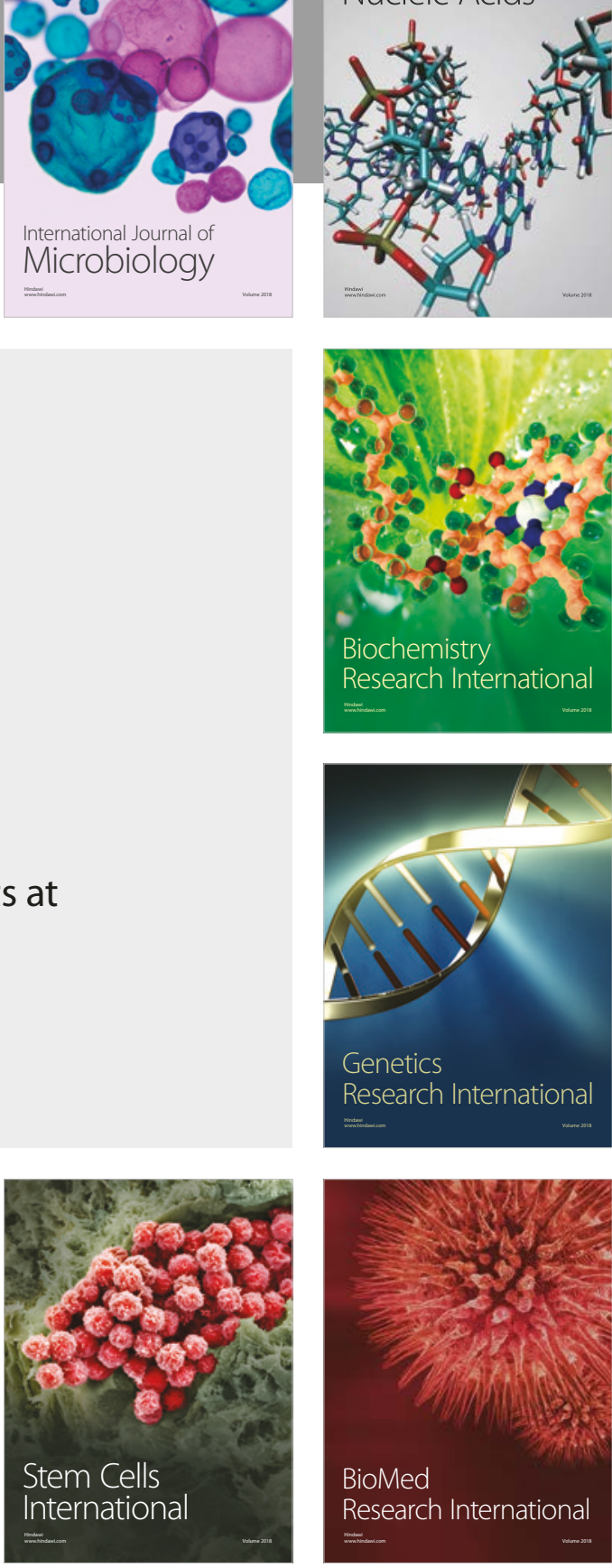
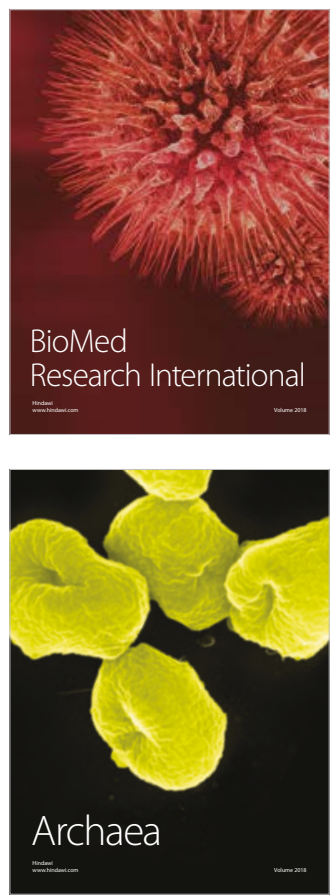